\title{
Safety and Efficacy of Indigenous Equine Antithymocyte Globulin Along with Cyclosporine in Subjects with Acquired Aplastic Anemia
}

\author{
M. B. Agarwal $\cdot$ Farah Jijina $\cdot$ Sandip Shah $\cdot$ \\ Pankaj Malhotra • Sharat Damodar • \\ Cecil Ross
}

Received: 14 January 2014 / Accepted: 10 June 2014/ Published online: 16 July 2014

(C) Indian Society of Haematology \& Transfusion Medicine 2014

\begin{abstract}
To confirm the safety and efficacy of an indigenous equine antithymocyte globulin (eATG) along with cyclosporine in Indian subjects with acquired aplastic anaemia. Subjects $>2$ years old with acquired aplastic anaemia were enrolled at six hospitals between April 2011 and February 2013, after approval from respective Ethics Committees. Equine ATG at a dose of $40 \mathrm{mg} / \mathrm{kg} / \mathrm{day}$ was infused for 4 days. Efficacy analysis defined a priori, was in subjects, who had completed eATG treatment and followed-up on day 90 and/or 180. Complete response (CR) was defined as-transfusion independent, haemoglobin $\geq 11 \mathrm{~g} / \mathrm{dL}$, absolute neutrophil count (ANC) $>1.510^{9} / \mathrm{L}$
\end{abstract}

\section{B. Agarwal ( $\square)$}

Hematology Centre, Ghamat Lodge, 2nd Floor, Above ING

Vaishya Bank, 804 A, Dr. B. Ambedkar Road, Dadar TT,

Mumbai, India

e-mail: mbagarwal14@gmail.com

F. Jijina

Department of Haematology, Seth GS Medical College \& KEM

Hospital, Ernest Borges Road, Parel, Mumbai, India

\section{S. Shah}

Hemato-Oncology Clinic, Vedanta Institute of Medical Sciences, 1st Floor, Stadium Commerce College Road, Navrangpura, Ahmedabad, India

\section{P. Malhotra}

Clinical Hematology, Department of Internal Medicine, Post Graduate Institute of Medical Education and Research, Sector-

12, Chandigarh, India

\section{S. Damodar}

Narayana Hrudayalaya, 258/A, Bommasandra Industrial Area, Anekal Taluk, Bangalore, India

C. Ross

Department of Haematology, St. John's Medical College,

Sarjapur Road, Bangalore, India and platelet $\geq 15010^{9} / \mathrm{L}$; partial response (PR) was transfusion independent, haemoglobin $\geq 8 \mathrm{~g} / \mathrm{dL}$, ANC $>0.510^{9} /$ $\mathrm{L}$ and platelet $\geq 2010^{9} / \mathrm{L}$; non responders were transfusion dependent. Lymphocyte subsets (CD 2, 3, 4 and 8) in the blood were tested on days 0 (pre eATG infusion), 3, 5, 7, 14 and 21 after eATG. Of the 30 subjects (two children $<12$ years old) enrolled, 19 completed day 90 and 18 completed day 180 visit. Of the remaining 11 subjects, two died on days 12 and 45 due to septicaemia and pneumonia, one was withdrawn after the first dose of eATG due to jaundice and eight were lost to follow-up. The median age was 30 (9-58) years and weight was $57(26-84) \mathrm{kg}$. On day 90, 12 of 30 subjects responded (CR 1, PR 11) and 15 of 30 (CR 2, PR 13) on day 180. The most common adverse event was fever related to eATG infusion. There were two serious adverse events (acute renal failure, febrile neutropenia) and both recovered with treatment. There were no unusual adverse events noted during the study period. Blood $\mathrm{T}$ lymphocytes showed a mean decrease of $91 \%$ from baseline that recovered by day 21 . We conclude that eATG is safe and in combination with cyclosporine showed overall response in $50 \%$ of enrolled subjects. The trial was registered with the clinical trial registry-india (Registration no. CTRI/2012/03/002498).

Keywords Immunosuppressive therapy - CD count . eATG $\cdot$ T lymphocyte

\section{Introduction}

Aplastic anemia (AA) is a hematopoietic stem cell disorder characterized by pancytopenia and hypocellular bone marrow in the absence of an abnormal infiltrate and no increase in reticulin. The incidence of acquired AA in 
Europe and North America is around 2 per million per year and two- to threefold higher in Asia [1, 2]. Hematopoietic stem cell transplant and immunosuppressive therapy (IST) are the primary modalities of treatment for AA. The efficacy of Immunosuppressive therapy (IST) in AA is well established since the 1970s, with several large prospective trials in the United States, Europe and Japan showing consistent results [3-5]. An effective IST regimen is equine anti-thymocyte globulin (eATG) in combination with cyclosporine which produces hematologic responses in $60-75 \%$ of cases $[1,6]$.

The present study was conducted to confirm the safety and efficacy of an indigenously developed eATG along with cyclosporine in Indian subjects with acquired aplastic anemia.

\section{Methods}

The indigenous eATG (Thymogam, manufactured by Bharat Serums and Vaccines Ltd. India.) was manufactured from healthy horses immunized with purified thymocytes obtained from children, below the age of 13 years, undergoing cardiac surgery. Plasmapheresis was carried out on horses showing appropriate titre of ATG, using lymphocytotoxicity assay. The hyperimmune plasma from several horses was pooled and fractionated using caprylic acid precipitation, followed by column chromatography, under GMP conditions to obtain highly purified $\operatorname{IgG}$ fraction, complying with the British Pharmacopoeia.

This phase IV, single group, prospective, multicentre study was conducted in subjects with acquired aplastic anemia of any severity at six centres across India between April 2011 and August 2013. The study protocol was approved by the institutional ethics committees at each centre.

Subjects aged above 2 years (children defined below 12 years of age), with acquired AA of any severity were eligible for enrolment. All subjects underwent standard investigations (complete hemogram, bone marrow aspiration and biopsy, liver and renal serum chemistries, plasma glucose, chest X-ray, electrocardiogram, hepatitis B, C and HIV serology and paroxysmal nocturnal hemoglobinuria). Subjects with Fanconi anemia (by chromosomal breakage studies), history of cancer, serum creatinine values more than 2.5 times upper limit of normal, seropositive for HIV, clonal disorders and pregnant women were excluded.

AA was defined as marrow cellularity of less than $25 \%$ of normal or less than $50 \%$ with haematopoietic cells representing less than $30 \%$ of the residual cells and at least two of the following peripheral blood counts: neutrophil count of less than $0.5 \times 10^{9} / \mathrm{L}$, platelet count of less than $20 \times 10^{9} / \mathrm{L}$, and reticulocyte count of less than $1 \%$ [6].
The disease severity was classified as non severe aplastic anaemia (NSAA) with $\mathrm{Hb}$ less than $10 \mathrm{~g} / \mathrm{dL}$, neutrophil count less than $1.5 \times 10^{9} / \mathrm{L}$ and platelet count less than $100 \times 10^{9} / \mathrm{L}$; severe aplastic anaemia (SAA) with 2 of the following neutrophil count less than $0.5 \times 10^{9} / \mathrm{L}$, platelet count less than $20 \times 10^{9} / \mathrm{L}$ and reticulocyte count less than $1 \%$ and very severe aplastic anaemia (VSAA) with absolute neutrophil count less than $0.2 \times 10^{9} / \mathrm{L}$ [7].

Enrolled subjects received eATG at a dose of $40 \mathrm{mg} / \mathrm{kg} /$ day for 4 days, administered as an IV infusion over $12 \mathrm{~h}$, after sensitivity testing with $0.1 \mathrm{ml}$ of eATG. Premedication, prophylactic antimicrobials and supportive care (blood and platelet transfusion) was provided as per individual hospital protocol. Cyclosporine (CSA) was administered at a dose of $4-6 \mathrm{mg} / \mathrm{kg} /$ day of eATG infusion to maintain trough levels of $200-400 \mathrm{ng} / \mathrm{mL}$ for 6 months duration of study. The actual dose given as well as the commencement or continuation of the CSA was as per investigator's discretion. There was no tapering of CSA during the study. Granulocyte Colony Stimulating Factor (G-CSF) was given after e ATG in 2 subjects: 1 (VSAA) on day 5, 6 and 7 showed partial response and 1 (SAA) on day 31 and 32 was non-responder. Subjects were called for follow-up on days 14, 21, 30, 45, 90 and 180 after first dose of eATG for clinical examination and laboratory investigations. End-of-study was on day 180 after first dose of eATG.

The trial was registered with the Clinical Trial RegistryIndia (Registration no. CTRI/2012/03/002498).

\section{Efficacy Assessment}

Response was graded as complete response (CR) or partial response (PR) as recommended by British Committee for Standards in Haematology (2009) guidelines (Table 1) [6]. Clinical examination and laboratory investigations (hemogram, liver and renal serum chemistries) were performed daily during eATG administration and then on each

Table 1 Response criteria in aplastic anemia according to British Committee for Standards in Haematology guidelines [7]

\begin{tabular}{ll}
\hline Response in aplastic anemia & Criteria \\
\hline Complete response & Transfusion independence \\
& $\mathrm{Hb} \geq 11 \mathrm{~g} / \mathrm{dL}$ \\
& Neutrophils $>1.5 \times 10^{9} / \mathrm{L}$ \\
& Platelets $>100 \times 10^{9} / \mathrm{L}$ \\
& Transfusion independence \\
Partial response & Hb $\geq 8 \mathrm{~g} / \mathrm{dL}$ \\
& Neutrophils $>0.5 \times 10^{9} / \mathrm{L}$ \\
& Platelets $>20 \times 10^{9} / \mathrm{L}$ \\
No response & Transfusion dependence \\
\hline
\end{tabular}


follow-up visit till day 180 end-of-study visit. Relapse was defined as reversal to transfusion dependence after partial or complete response.

Subjects blood was also analysed for $\mathrm{T}$ lymphocyte subsets on pre eATG infusion (day 0 ) and on days 3, 5, 7, 14 and 21 after first dose of eATG at a centralized lab. Blood samples were collected from the sites and couriered to the central laboratory within $8 \mathrm{~h}$ for testing. Counts for $\mathrm{CD} 2, \mathrm{CD} 3, \mathrm{CD} 4$ and $\mathrm{CD} 8$ were determined by Flow Cytometry (FACS Calibur; Becton-Dickinson) with immunofluorescent monoclonal antibodies (Phycoerythrin Mouse Anti Human CD2, Fluorescein Isothiocyanate Mouse Anti Human CD4, Fluorescein Isothiocyanate Mouse Anti Human CD3, Fluorescein Isothiocyanate Mouse Anti Human CD8; Becton-Dickinson). $100 \mu 1$ blood was incubated with $10 \mu \mathrm{l}$ of the Fluorescent Antibody at room temperature for $15 \mathrm{~min}$ in the dark. $2 \mathrm{ml}$ of $1 \times$ Lysing solution (BD FACS Lysing Solution 10×; Becton-Dickinson) was added to each tube and the same was incubated at room temperature for $15 \mathrm{~min}$ in the dark. Tubes were spun at $1500 \mathrm{rpm}$ for $5 \mathrm{~min}$, after which the supernatant was discarded and the tubes were washed once with $2 \mathrm{ml}$ of Sheath Fluid (BD FACS Flow; BectonDickinson). The cells were re-suspended in $300 \mu \mathrm{l}$ of Sheath Fluid and 10,000 events were read on the flow cytometer. CD counts were analysed by comparing the percent gated cells with the absolute lymphocyte count.

\section{Safety Assessment}

All subjects were regularly monitored for adverse events (AE) till completion of study. Infusion related events were monitored during eATG infusion daily. Adverse events were assessed for their seriousness and their relationship to eATG.

\section{Statistical Analysis}

The analysis of data was performed by an independent biostatistician. For categorical data, frequency counts (n) and percentage (\%) was presented. Changes in subject variables throughout the study duration are presented as median with further analysis using repeated measure ANOVA test.

Results

Of the 30 subjects recruited, 20 were male. The median (range) age of the subjects was 30 (9-58) years and weight was $57(26-84) \mathrm{kg}$. The median (range) duration from diagnosis of AA to start of eATG administration was 3 (1-24) months. Eight subjects had non-severe aplastic anemia (NSAA), 17 had severe aplastic anemia (SAA) and five had very severe aplastic anemia (VSAA).
Table 2 Response to eATG with cyclosporine according to severity of aplastic anemia

\begin{tabular}{|c|c|c|c|c|c|c|c|}
\hline \multirow{2}{*}{$\begin{array}{l}\text { Severity } \\
\text { of aplastic } \\
\text { anemia }\end{array}$} & \multirow[t]{2}{*}{$\mathrm{n}(\%)$} & \multicolumn{3}{|c|}{$\leq$ Day 90} & \multicolumn{3}{|c|}{ Day 180} \\
\hline & & $\begin{array}{l}\text { CR } \\
\text { n }(\%)\end{array}$ & $\begin{array}{l}\mathrm{PR} \\
\mathrm{n}(\%)\end{array}$ & $\begin{array}{l}\text { NR } \\
\text { n }(\%)\end{array}$ & $\begin{array}{l}\text { CR } \\
\text { n (\%) }\end{array}$ & $\begin{array}{l}\mathrm{PR} \\
\mathrm{n}(\%)\end{array}$ & $\begin{array}{l}\text { NR } \\
\text { n }(\%)\end{array}$ \\
\hline NSAA & $\begin{array}{l}8 \\
(26.6)\end{array}$ & $\begin{array}{l}1 \\
(12.5)\end{array}$ & $\begin{array}{l}4 \\
(50)\end{array}$ & $\begin{array}{l}3 \\
(37.5)\end{array}$ & $\begin{array}{l}2 \\
(25)\end{array}$ & $\begin{array}{l}4 \\
(50)\end{array}$ & $\begin{array}{l}2 \\
(25)\end{array}$ \\
\hline SAA & $\begin{array}{l}17 \\
(56.6)\end{array}$ & 0 & $\begin{array}{l}6 \\
(35.3)\end{array}$ & $\begin{array}{l}11 \\
(64.7)\end{array}$ & 0 & $\begin{array}{l}7 \\
(41.1)\end{array}$ & $\begin{array}{l}10 \\
(58.8)\end{array}$ \\
\hline VSAA & $\begin{array}{l}5 \\
(16.6)\end{array}$ & 0 & $\begin{array}{l}1 \\
(20)\end{array}$ & $\begin{array}{l}4 \\
(80)\end{array}$ & 0 & $\begin{array}{l}2 \\
(40)\end{array}$ & $\begin{array}{l}3 \\
(60)\end{array}$ \\
\hline
\end{tabular}

$N R$ non-responders, $C R$ complete responders, $P R$ partial responders, NSAA non-severe aplastic anemia, SAA severe aplastic anemia, VSAA very severe aplastic anemia

Twenty-eight subjects received complete four doses of eATG. One subject developed jaundice after first dose of eATG and was withdrawn from the study by the investigator. One subject had an adverse event (acute renal failure) on day 3 hence the $4^{\text {th }}$ day dose was not given; this subject completed day 180 follow up. Nineteen subjects followed up till day 90 and 18 till day 180 (one subject lost to follow up post day 90). Among those who did not complete day 90 follow up, two subjects died (on day 12 and 45) and eight were lost to follow-up. These eight patients were discharged and did not return for subsequent follow-ups: two of them withdrew consent telephonically and six were not reachable despite repeated attempts by telephone and by study co-ordinator visits to the address given by the patients, from where they had moved without any forwarding address available.

At 90 day follow-up, one subject had complete response (CR) and 11 subjects showed partial response (PR) (total response-12 of 30). At 180 days, two subjects had CR and 13 had PR (total response-15 of 30). The response to treatment in subjects with NSAA, SAA and VSAA is summarized in Table 2. This included two children; one child with SAA showed partial response on day 180 and one VSAA was lost to follow up after completing 4 days of eATG treatment.

The mean dose of CSA prescribed to subjects enrolled in the study was $4.5 \mathrm{mg} / \mathrm{kg}$ body weight. The median (range) was 3.23 (1.9-6.6). The decision on actual dose was left to the investigator's discretion. Time to commencement of CSA as advised in the protocol was 2 weeks after last dose of ATG but five of 29 patients were already on CSA prior to enrollment in the study and this was not discontinued; in three patients CSA was commenced within 10 days after eATG administration. Balance 21 patients commenced CSA 2 weeks after last dose of eATG. 28 of 30 patients were given premedication with steroids during the 4 days 


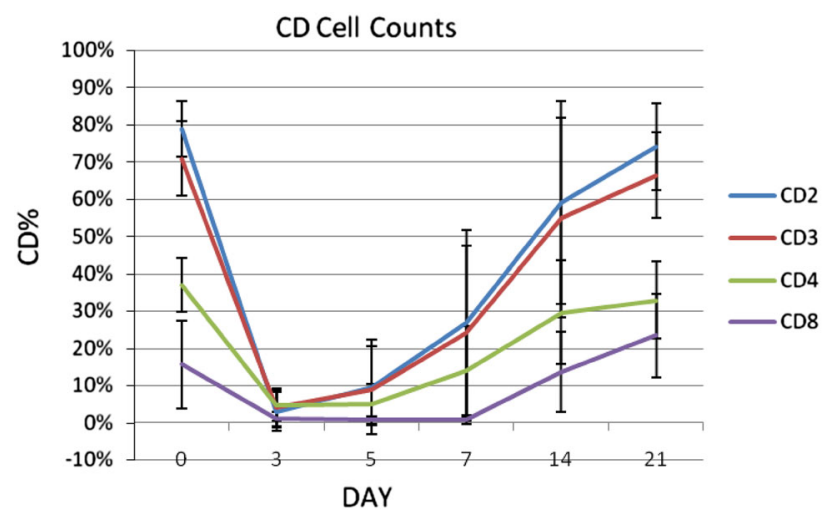

Fig. 1 Blood T lymphocyte counts on days 0 (pre eATG), 3, 5, 7, 14 and 21 after eATG administration

of eATG administration (four subjects each received methylprednisolone and dexamethasone and 20 subjects received hydrocortisone). For serum sickness prophylaxis, the patients were given oral prednisone $1 \mathrm{mg} / \mathrm{kg} /$ day prior to the first dose of ATG and continued for 10 days and then tapered over the subsequent 7 days. Antimicrobial prophylaxis was given as per hospital practice to the patients -25 of the 30 subjects received antibacterial; 19 of these 25 received antifungal and seven also received antiviral prophylaxis in addition. Therapeutic antibiotics were administered to five patients for eight events (fever (five) and one each of abscess/urinary tract infection/gingivitis).

G-CSF was given after e ATG in two patients; one patient (VSAA) received G-CSF on days 5-7 after commencement of eATG and showed partial response and one patient (SAA) received G-CSF on days 31 and 32 but did not respond to treatment.

The median duration between diagnosis of AA and institution of IST was 3 (1-24) months in the responders and 10 (3-10) months in the non-responders. The blood $\mathrm{T}$ cell subset counts decreased by $91 \%$ by day 5 and recovered completely by day 21 in all subjects (Fig. 1).

A total of 79 non-serious adverse events were reported of which 28 were eATG related and 51 were unrelated. (Table 3). Of these, 26 occurred during eATG infusion (16 related); 53 occurred post eATG infusion (12 related). Fever (15 subjects) was the most common adverse event reported followed by vomiting (five subjects). Anorexia (three subjects) was seen only post eATG administration. All these non-serious adverse events recovered without any sequelae.

Serious adverse events were noted in five subjects. One subject developed acute renal failure on day 3 which resolved completely by day 30 after receiving standard treatment. One subject was readmitted for febrile neutropenia on day 30 for 3 days and discharged on recovery. One subject had seizure with no apparent cause and recovered. There were two deaths; one subject died at home on day 45 due to unresolved pneumonia and one subject died on day 12 due to septicemia.

\section{Discussion}

We assessed the safety and efficacy of indigenously developed eATG in 30 Indian subjects with acquired aplastic anemia at six centres across India. Eight subjects were lost to follow up mainly because many changed their address or moved to hometowns in remote places and did not adhere to follow up visit dates. This, along with two cases of early deaths, one withdrawn after first dose of eATG, left 19 subjects who completed day 90 follow up and 18 subjects with day 180 follow-up (one lost to followup after day 90).

For ITT analysis, the subjects who were lost to follow-up were considered as non responders. ITT analysis revealed a response in 12 of 30 subjects ( $40 \%$ ) at or before day 90 and 15 of $30(50 \%)$ at day 180 post eATG administration along with cyclosporin. The total number of subjects was small, but the results are in accordance with earlier studies which have reported response rates of 33-85\% [3, 5, 8-16]. The findings of various Indian studies have been summarised in Table 4 . The response rate of $50 \%$ in our study is distributed across all severity groups supporting the use of eATG in subjects with AA of any severity.

ATG is a polyclonal antibody that recognises various antigens on the cell surface membranes and is cytotoxic to lymphocytes in vitro. The immunosuppressive effect is mediated through lymphocyte depletion, modulation or coating. ATG is reported to have activity against CD2, CD3, CD4, CD5, CD6, CD7, CD8, CD11a, CD16, CD18, CD28, CD38, CD40, CD44 and CD 45 and T cell receptors $\alpha / \beta$ antigens. $C D 2, C D 3$ and $C D 4$ and CD 8 counts tested in our study were seen to fall between days 3 and 5 after eATG administration and recover between days 7 and 14 . This confirms the action of eATG on T cell-mediated immunity and in our study this result was seen in every patient irrespective of the clinical response on day 180.

The duration between diagnosis of AA and institution of IST was greater in the non-responders than in the responders. This emphasizes the need to start therapy early in the course of the disease. Eighteen of $30(60 \%)$ of those treated with eATG were still surviving at the last postprotocol follow-up (November 2013).

A total of five serious adverse events were reported, including two deaths. Both the deaths resulted from infective pathology (pneumonia and septicemia). The three other SAEs-acute renal failure, febrile neutropenia and seizures resolved with medical management. All the 79 non-serious adverse events reported in the study resolved with supportive care and there were no unknown adverse 
Table 3 Non serious adverse events during and post-eATG administration

\begin{tabular}{|c|c|c|c|c|c|c|}
\hline \multirow[t]{2}{*}{ Sr. No. } & \multirow[t]{2}{*}{ Category } & \multirow[t]{2}{*}{ Adverse event } & \multicolumn{2}{|c|}{$\begin{array}{l}\text { During eATG } \\
\text { Infusion } n \\
\text { (subject nos) }\end{array}$} & \multicolumn{2}{|c|}{$\begin{array}{l}\text { Post eATG Infusion } \\
\mathrm{n} \text { (subject nos) }\end{array}$} \\
\hline & & & Related & Unrelated & Related & Unrelated \\
\hline 1 & General/systemic & Allergic urticaria & 2 & - & - & 2 \\
\hline 2 & & Bodyache & - & - & - & 2 \\
\hline 3 & & Erythema & - & - & - & 1 \\
\hline 4 & & Fever & 6 & 5 & - & 8 \\
\hline 5 & & Serum sickness & - & - & 1 & - \\
\hline 6 & & Swelling on the face & - & - & 1 & - \\
\hline 7 & & Weakness & - & - & - & 1 \\
\hline 8 & & Weight gain & - & - & 1 & - \\
\hline 9 & & Acute parotitis & - & - & - & 1 \\
\hline 10 & & Anorexia & - & - & - & 3 \\
\hline 11 & & Constipation & - & - & - & 2 \\
\hline 12 & & Gingivitis & - & - & 1 & - \\
\hline 13 & & Mucositis & - & - & - & 1 \\
\hline 14 & & Oral ulcer & - & - & - & 2 \\
\hline 15 & & Acidity & - & 1 & - & - \\
\hline 16 & & Vomiting & 2 & 1 & - & 2 \\
\hline 17 & & Per vaginal bleeding & - & - & - & 1 \\
\hline 18 & & Oral bleeding & - & - & - & 1 \\
\hline 19 & & Mild per rectal bleeding & - & - & - & 1 \\
\hline 20 & Renal system & Acute renal failure & 1 & - & - & - \\
\hline 21 & & Urinary tract infection & - & - & - & 2 \\
\hline 22 & Biochemical & Deranged LFT & 1 & - & 1 & - \\
\hline 23 & & Kidney dysfunction & 1 & - & 1 & - \\
\hline 24 & Extremities & Bilateral pedal oedema & - & - & 2 & - \\
\hline 25 & & Joint pain & - & - & 2 & - \\
\hline 26 & Respiratory system & Bilateral pleural effusion & - & - & 1 & - \\
\hline 27 & & Dyspnea & - & - & - & 1 \\
\hline 28 & & Cough & - & - & - & 1 \\
\hline 29 & & Pain in nostrils & - & - & - & 1 \\
\hline 30 & Ophthalmic & Blurred vision & - & 1 & - & - \\
\hline 31 & & Burning of eye & - & - & 1 & - \\
\hline 32 & CNS & Shivering & - & - & 1 & - \\
\hline 33 & & Headache & - & - & - & 2 \\
\hline 34 & Dermatologic & Ichthyosis & - & - & - & 1 \\
\hline 35 & & Abscess in neck & 1 & - & - & - \\
\hline 36 & & Papular rash/patches & 1 & 1 & - & 2 \\
\hline 37 & CVS & Thrombocytopenia & - & - & - & 1 \\
\hline 38 & & Elevated serum Ferritin levels & - & - & - & 2 \\
\hline 39 & Endocrine & Hyperglycemia & - & 1 & - & - \\
\hline Total & & & 16 & 10 & 12 & 41 \\
\hline
\end{tabular}

effects. Some of the adverse events may be related to administration of corticosteroids and cyclosporine or due to basic disease and not necessarily related to eATG administration.
In conclusion, this study demonstrates that immunosuppression with eATG developed indigenously by Bharat Serum and Vaccine Ltd, India, in combination with cyclosporine, is safe and effective first line therapy in 
Table 4 Summary of response to antithymocyte/ antilymphocyte globulin with or without cyclosporine in Indian studies

\begin{tabular}{|c|c|c|c|c|c|c|}
\hline Study & Drug used & Design & $\begin{array}{l}\text { Age group } \\
\text { (years) }\end{array}$ & $\begin{array}{l}\mathrm{n} \\
\text { (total) }\end{array}$ & Responders & $\begin{array}{l}\text { Relapse } \\
(\%)\end{array}$ \\
\hline Gupta et al. [15] & Thymogam & Retrospective & $4-14$ & 30 & $\begin{array}{r}33.3 \% \text { at } \\
6 \text { month }\end{array}$ & 3 \\
\hline Sharma et al. [16] & $\begin{array}{l}\text { Atgam/Thymogam/ } \\
\text { Lymphoglobuline }\end{array}$ & Retrospective & $5-12$ & 35 & $50 \%$ at $1 \mathrm{yr}$ & 19 \\
\hline Chandra et al. [17] & $\begin{array}{l}\text { Atgam/Thymogam/ } \\
\text { Lymphoglobuline }\end{array}$ & Prospective & $6-12$ & 23 & $\begin{array}{c}40 \% \text { at } \\
6 \text { month } \\
45 \% \text { at } \\
1 \text { year }\end{array}$ & 10 \\
\hline Nair et al. [18] & Atgam & Prospective & $7-18$ & 33 & $\begin{array}{r}87.9 \% \text { at } \\
6 \text { month }\end{array}$ & 3 \\
\hline Agarwal et al. [19] & Atgam/ALG & Prospective & $12-63$ & 35 & $\begin{array}{l}54.3 \% \text { after } \\
6 \text { month }\end{array}$ & 3 \\
\hline Current study & Thymogam & Prospective & $9-58$ & 30 & $\begin{array}{l}40 \% \text { at } \\
3 \text { months } \\
50 \% \text { at } \\
6 \text { months }\end{array}$ & - \\
\hline
\end{tabular}

subjects with AA who are unsuitable for hematopoietic stem cell transplantation.

Acknowledgments This study was funded by Bharat Serums and Vaccines Limited, India who provided the study medications, administrative assistance and study grants to participating centres

Ethical Standards This study has been approved by the Institutional ethics committees at all centres and has been performed in accordance with the ethical standards laid down in the 1964 Declaration of Helsinki and its later amendments. All subjects enrolled in the study gave their informed consent prior to their inclusion in the study.

\section{References}

1. Young NS, Calado RT, Scheinberg P (2006) Current concepts in the pathophysiology and treatment of aplastic anemia. Blood 108(8):2509-2519

2. Sharma S, Malhotra P, Lal V, Singh P, Varma N, Varma S (2012) Asymptomatic cerebral bleeds in patients with aplastic anemia. Ann Hematol 91(8):1187-1191

3. Scheinberg P, Nunez O, Weinstein B et al (2011) Horse versus rabbit antithymocyte globulin in acquired aplastic anemia. N Engl J Med 365(5):430-438

4. Teramura M, Kimura A, Iwase S, Yonemura Y, Nakao S, Urabe A et al (2007) Treatment of severe aplastic anemia with antithymocyte globulin and cyclosporin A with or without G-CSF in adults: a multicenter randomized study in Japan. Blood 110: 1756-1761

5. Bacigalupo A, Bruno B, Saracco P et al (2000) Antilymphocyte globulin, cyclosporine, prednisolone, and granulocyte colonystimulating factor for severe aplastic anemia: an update of the GITMO/EBMT study on 100 patients: European Group for Blood and Marrow Transplantation (EBMT) Working Party on Severe Aplastic Anemia and the Gruppo Italiano Trapianti di Midolio Osseo (GITMO). Blood 95:1931-1934
6. Frickhofen N, Heimpel H, Kaltwasser JP, Schrezenmeier H (2003) Antithymocyte globulin with or without cyclosporin A: 11-year follow- up of a randomized trial comparing treatments of aplastic anemia. Blood 101(4):1236-1242

7. Marsh JCW, Ball SE, Cavenagh J, British Committee for Standards in Haematology et al (2009) Guidelines for the diagnosis and management of aplastic anemia. Br J Haematol 147:43-70

8. Rosenfeld S, Follmann D, Nunez O, Young NS (2003) Antithymocyte globulin and cyclosporine for severe aplastic anemia: association between hematologic response and long-term outcome. JAMA 289(9):1130-1135

9. Scheinberg P, Wu CO, Nunez O, Scheinberg P, Boss C, Sloand EM et al (2009) Treatment of severe aplastic anemia with a combination of horse antithymocyte globulin and cyclosporine, with or without sirolimus: a prospective randomized study. Haematologica 94(3):348-354

10. Rosenfeld SJ, Kimball J, Vining D, Young NS (1995) Intensive immunosuppression with antithymocyte globulin and cyclosporine as treatment for severe acquired aplastic anemia. Blood 85(11):3058-3065

11. Gupta V, Kumar A, Tilak V, Saini I, Bhatia B (2012) Immunosuppressive therapy in aplastic anemia. Indian J Pediatr 79(12): 1587-1591

12. Sharma R, Chandra J, Sharma S, Pemde H, Singh V (2012) Antithymocyte globulin and cyclosporine in children with aplastic anemia: a developing country experience. J Pediatr Hematol Oncol 34:93-95

13. Chandra J, Naithani R, Ravi R, Singh V, Narayan S, Sharma S et al (2008) Antithymocyte globulin and cyclosporine in children with acquired aplastic anemia. Indian J Pediatr 75(3):229-233

14. Nair V, Sondhi V, Sharma A, Das S, Sharma S (2012) Survival after immunosuppressive therapy in children with aplastic anemia. Indian Pediatr 49:371-376

15. Agarwal MB, Agarwal UM, Bhave AB, Vishwanathan C (1993) Anti-lymphocyte globulin therapy in acquired aplastic anemia. J Assoc Physicians India 41:371-373

16. Kojima S, Hibi S, Kosaka Y, Yamamoto M, Tsuchida M, Mugishima $\mathrm{H}$ et al (2000) Immunosuppressive therapy using antithymocyte globulin, cyclosporine, and danazol with or without human granulocyte colony-stimulating factor in children with acquired aplastic anemia. Blood 96(6):2049-2054 